\title{
Drag-out effect of piezomagnetic signals due to a borehole: the Mogi source as an example
}

\author{
Yoichi Sasai $\left({ }^{1}\right)$, Malcom J.S. Johnston $\left({ }^{2}\right)$, Yoshikazu Tanaka $\left(^{3}\right)$, Robert Mueller $\left({ }^{2}\right)$, Takeshi Hashimoto $\left({ }^{4}\right)$, \\ Mitsuru Utsugi $\left({ }^{3}\right)$, Shinya Sakanaka $\left({ }^{5}\right)$, Makoto Uyeshima $\left({ }^{6}\right)$, Jacques Zlotnicki $\left({ }^{7}\right)$ and Paul Yvetot $\left({ }^{7}\right)$ \\ $\left.{ }^{(}\right)$Disaster Prevention Division, Bureau of General Affairs, Tokyo Metropolitan Government, Tokyo, Japan \\ ${ }^{(2)}$ US Geological Survey, Menlo Park, CA, U.S.A. \\ (3) Graduate School of Science, Kyoto University, Japan \\ $\left({ }^{4}\right)$ Graduate School of Science, Hokkaido University, Japan \\ $\left(^{5}\right)$ Faculty of Engineering and Resource Science, Akita University, Japan \\ $\left({ }^{6}\right)$ Earthquake Research Institute, The University of Tokyo, Japan \\ ${ }^{(7)}$ Observatoire de Physique du Globe de Clermont-Ferrand, France
}

\begin{abstract}
We show that using borehole measurements in tectonomagnetic experiments allows enhancement of the observed signals. New magnetic dipoles, which vary with stress changes from mechanical sources, are produced on the walls of the borehole. We evaluate such an effect quantitatively. First we formulate a general expression for the borehole effect due to any arbitrary source models. This is valid everywhere above the ground surface as well as within the cylindrical hole. A first-order approximate solution is given by a line of horizontal dipoles and vertical quadrupoles along the central axis of the borehole, which is valid above the ground surface and a slightly away (several tens of $\mathrm{cm}$ ) from the top of the borehole. Selecting the Mogi model as an example, we numerically evaluated the borehole effect. It turned out that the vertical quadrupoles produce two orders of magnitude more intense magnetic field than the horizontal dipoles. The borehole effect is very local, i.e. detectable only within a few $\mathrm{m}$ from its outlet, since it is of the same order or more than the case without a borehole. However, magnetic lines of force cannot reach the ground surface from a deeper portion $(>10 \mathrm{~m})$ of a borehole.
\end{abstract}

Key words piezomagnetic effect - borehole magnetic measurement - the Mogi model - Long Valley Caldera

\section{Introduction}

Boreholes are now widely used in geophysical observations, in particular for seismic and

Mailing address: Dr. Yoichi Sasai, Disaster Prevention Division, Bureau of General Affairs, Tokyo Metropolitan Government, Nishi-Shinjuku 2-8-1, Shinjuku-ku, Tokyo, 163-8001, Japan; e-mail: yosasai@zag.att.ne.jp crustal strain measurements, since they greatly reduce noise resulting from artificial mechanical vibrations and increase tectonic signals as the sensor is closer to the source. Improvement of $\mathrm{S} / \mathrm{N}$ ratio by the use of a borehole can be similarly expected in the case of tectonomagnetic observations. To avoid cultural noises due to moving magnetic bodies (cars, etc.), high-powered electric devices and so on, we must isolate an undisturbed area of at least a few hundreds meters square. Under the ground, however, high-frequency EM noise is almost completely shielded and a moving car is not detected at a $200 \mathrm{~m}$ depth (Yamamoto, 1990). Furthermore, tectonomagnetic signals increase with depth. 
However, we need special considerations in the case of magnetic field observations. We make measurements within the source material of the magnetized crust. When we excavate a hole, additional magnetic poles are observed along its wall. When a magnetometer is placed under the ground, the effect of the shallower magnetic source is different from the on-the-ground observation in which the source always lies beneath the sensor. Sasai (1994) evaluated both the effects in the case of a vertical rectangular strike-slip fault, and demonstrated that piezomagnetic signals are significantly enhanced at depth beneath the ground surface.

The underground solution for the piezomagnetic field is defined as a limit of the field value when the radius of the excavated spherical cavity approaches zero (Sasai, 1991b). We assume that the drilled hole is filled up with the same magnetic substance. In actual situations, a borehole would rarely be buried again, nor could the spherical cavity for a magnetometer ever be shrunk to zero volume. Usually, a casing-pipe is inserted to protect the borehole, in which a non-magnetic tube (e.g., vinyl chloride) is preferable, at least for shallow boreholes. This is because long-term alteration of magnetic susceptibility in high $\mu$ metals could disturb tectonomagnetic changes.

When we consider such a tectonomagnetic observation system, the existence of a borehole itself could produce an additional magnetic signal. As will be described later, we observed an interesting magnetic change associated with a volcano-tectonic event in Long Valley Caldera, California, U.S.A. We put two proton magnetometers only $40 \mathrm{~m}$ apart, one of which is located close to a buried borehole. The simple difference between the two magnetometers showed a variation more or less similar to the strain change at the observation site. A possibility was that amplified tectonomagnetic signal resulted from the presence of the borehole. We will examine quantitatively how much local magnetic field will be produced on the ground simply by the existence of a borehole.

\section{Theory}

We assume that the Earth is a homogeneous and isotropic elastic half-space with a uniformly magnetized top layer stressed by some pressure source. Suppose that there is a vertical cylindrical borehole with radius $a$ and length $L$ as shown in fig. 1. For the sake of simplicity, the borehole is located outside the pressure source so that no mechanical singularity due to the source exists along the surface of the borehole. As a result of crustal stress, magnetic poles resulting from piezomagnetism of rocks appear on the wall of the borehole. Given the displacement field due to the source, we can evaluate the influence of a borehole.

The piezomagnetic potential $W_{k}\left(\boldsymbol{r}_{0}\right)$ of a stressed magnetoelastic body with its surface $S$ and the displacement field $u_{k}(\boldsymbol{r})$ can be expressed as (Sasai, 1983, 1991b)

$$
\begin{aligned}
& W_{k}\left(\boldsymbol{r}_{0}\right)=C_{k} \iint_{S}\left[\left\{-\frac{\partial u_{k}(\boldsymbol{r})}{\partial n}+\frac{2(\lambda+\mu)}{3 \lambda+2 \mu} \Delta \boldsymbol{m}^{(k)} \cdot \boldsymbol{n}\right\}\right. \\
& \left.\frac{1}{\rho}+\left\{u_{k}(\boldsymbol{r})\right\} \frac{\partial}{\partial n}\left(\frac{1}{\rho}\right)\right] \mathrm{d} S
\end{aligned}
$$

where

$$
\Delta \boldsymbol{m}^{(k)}=\frac{3}{2}\left(\frac{\partial u_{k}}{\partial x_{l}}+\frac{\partial u_{l}}{\partial x_{k}}\right)-\delta_{k l} \operatorname{div} \boldsymbol{u} .
$$

In the above, $k$ indicates the $k$-th Cartesian component, $\boldsymbol{n}$ an outward normal to the surface $S, \boldsymbol{r}_{0}$ the observation point, $\boldsymbol{r}$ an arbitrary point on $S$ and $\rho=\left|\boldsymbol{r}-\boldsymbol{r}_{0}\right| . C_{k}$ is given by

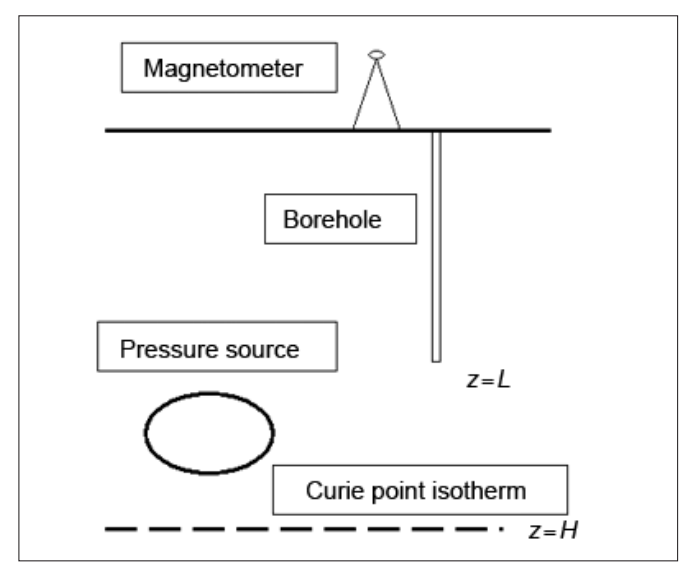

Fig. 1. Schematic representation of a borehole, a pressure source and the magnetized crust. 


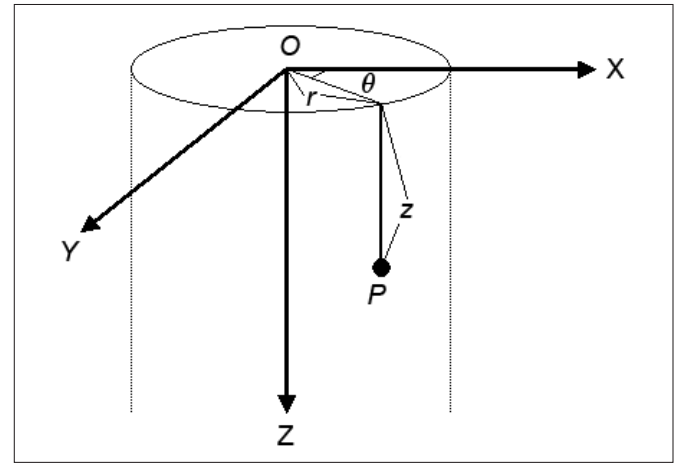

Fig. 2. The Cartesian and cylindrical coordinate system used in Section 2.

$$
C_{k}=\frac{1}{2} \beta J_{k} \mu \frac{3 \lambda+2 \mu}{\lambda+\mu}
$$

in which $\beta$ is the stress sensitivity, $J_{k} k$-th component of the magnetization, $\lambda$ and $\mu$ Lame's constants, and in particular $\mu$ the rigidity.

Let us consider the local effect of the borehole above the ground surface near around its top. The piezomagnetic potential due to the borehole $W_{k}^{(B H)}$ consists of three parts

$$
W_{k}^{(B H)}=W_{k}^{(A)}+W_{k}^{(B)}-W_{k}^{(C)}
$$

$W_{k}^{(A)}$ is the potential produced by magnetic poles along the cylindrical wall, $W_{k}^{(B)}$ by those over the circular bottom, and $W_{k}^{(C)}$ by those from the vanishing portion of the ground surface, respectively. We will evaluate these terms separately. First we introduce the Cartesian coordinates with $x-y$ plane on the Earth's surface and $z$ axis positive downward, of which origin is at the center of the borehole. We also define the cylindrical coordinates system which shares the origin and $z$-axis with the Cartesian one as shown in fig. 2.

\subsection{The contribution from the cylindrical wall of the borehole: $\mathrm{W}_{\mathrm{k}}{ }^{(\mathrm{A})}$}

Let the observation point outside the magnetoelastic body be $Q\left(x_{0}, y_{0}, z_{0}\right)=Q\left(r_{0}, \theta_{0}, z_{0}\right)$ and a moving point on the cylindrical surface of the borehole $P(x, y, z)=P(r, \theta, z)$. We find

$$
\begin{gathered}
\rho=\overline{P Q}=\sqrt{r_{0}^{2}+a^{2}+\left(z-z_{0}\right)^{2}-2 a r_{0} \cos \left(\theta-\theta_{0}\right)} \\
\boldsymbol{n}=-\mathbf{e}_{r}=(-\cos \theta,-\sin \theta, 0) .
\end{gathered}
$$

The integral over the cylindrical surface is given by

$$
\begin{aligned}
& \frac{1}{C_{k}} W_{k}^{(A)}\left(\boldsymbol{r}_{0}\right)=a \int_{0}^{L} \mathrm{~d} z\left[\int_{0}^{2 \pi} F_{A}^{(k)} \frac{\cos \theta}{\rho} \mathrm{d} \theta+\right. \\
& \left.+\int_{0}^{2 \pi} F_{B}^{(k)} \frac{\sin \theta}{\rho} \mathrm{d} \theta+\int_{0}^{2 \pi} F_{C}^{(k)} \frac{-a+r_{0} \cos \left(\theta-\theta_{0}\right)}{\rho^{3}} \mathrm{~d} \theta\right]
\end{aligned}
$$

where

$$
\begin{gathered}
F_{A}^{(k)}=\left[-\frac{\mu}{3 \lambda+2 \mu} \frac{\partial u_{k}}{\partial x}-\frac{3(\lambda+\mu)}{3 \lambda+2 \mu} \frac{\partial u_{x}}{\partial x_{k}}+\right. \\
\left.+\frac{2(\lambda+\mu)}{3 \lambda+2 \mu} \delta_{k x} \operatorname{div} \boldsymbol{u}\right]_{r=a} \\
F_{B}^{(k)}=\left[-\frac{\mu}{3 \lambda+2 \mu} \frac{\partial u_{k}}{\partial y}-\frac{3(\lambda+\mu)}{3 \lambda+2 \mu} \frac{\partial u_{y}}{\partial x_{k}}+\right. \\
\left.+\frac{2(\lambda+\mu)}{3 \lambda+2 \mu} \delta_{k y} \operatorname{div} \boldsymbol{u}\right]_{r=a} \\
F_{C}^{(k)}=\left[u_{k}\right]_{r=a} .
\end{gathered}
$$

Since the radius of the borehole $a$ is sufficiently small, the displacement at an arbitrary depth $z$ is almost identical along the wall surface. It can be replaced with the value at the central axis $r=0$, which could be attained if the borehole were absent. $F_{A}{ }^{(k)}, F_{B}{ }^{(k)}$ and $F_{C}{ }^{(k)}$ are functions of $z$ only, which are given by

$$
\begin{gathered}
F_{A}^{(k)}(z)=\left[-\frac{\mu}{3 \lambda+2 \mu} \frac{\partial u_{k}}{\partial x}-\frac{3(\lambda+\mu)}{3 \lambda+2 \mu} \frac{\partial u_{x}}{\partial x_{k}}+\right. \\
\left.+\frac{2(\lambda+\mu)}{3 \lambda+2 \mu} \delta_{k x} \operatorname{div} \boldsymbol{u}\right]_{r=0} \\
F_{B}^{(k)}(z)=\left[-\frac{\mu}{3 \lambda+2 \mu} \frac{\partial u_{k}}{\partial y}-\frac{3(\lambda+\mu)}{3 \lambda+2 \mu} \frac{\partial u_{y}}{\partial x_{k}}+\right. \\
\left.+\frac{2(\lambda+\mu)}{3 \lambda+2 \mu} \delta_{k y} \operatorname{div} \boldsymbol{u}\right]_{r=0} \\
F_{C}^{(k)}(z)=\left[u_{k}\right]_{r=0}
\end{gathered}
$$


These terms are excluded from the integrals with respect to $\theta$, and eq. (2.7) is reduced to

$$
\begin{aligned}
& \frac{1}{C_{k}} W_{k}^{(A)}\left(\boldsymbol{r}_{0}\right)=2 a \int_{0}^{L} \mathrm{~d} z\left[F_{A}^{(k)} \cos \theta_{0} \Phi_{2}+F_{B}^{(k)} \sin \theta_{0} \Phi_{2}+\right. \\
& \left.+F_{C}^{(k)}\left(a \Phi_{3}-r_{0} \Phi_{4}\right)\right]
\end{aligned}
$$

where

$$
\begin{aligned}
& \Phi_{2}=\int_{0}^{\pi} \frac{\cos \phi}{\rho_{\phi}} \mathrm{d} \phi=\pi I(1,1 ; 0) \\
& \Phi_{3}=\int_{0}^{\pi} \frac{\cos \phi}{\rho_{\phi}^{3}} \mathrm{~d} \phi=\frac{\pi}{c} I(0,0 ; 1) \\
& \Phi_{4}=\int_{0}^{\pi} \frac{\cos \phi}{\rho_{\phi}^{3}} \mathrm{~d} \phi=\frac{\pi}{c} I(1,1 ; 1)
\end{aligned}
$$

in which

$$
\rho_{\phi}=\sqrt{R^{2}+c^{2}}
$$

and

$$
R=\sqrt{a^{2}-2 a r_{0} \cos \phi+r_{0}^{2}}, \quad c=\left|z-z_{0}\right|
$$

$I(m, n ; l)$ 's on the righthand side of eqs. ((2.15) to (2.17)) are called the integrals of LipschitzHankel type, whose characteristics are investigated in detail by Eason et al. (1955)

$$
I(m, n ; l)=\int_{0}^{\infty} J_{m}(a t) J_{n}\left(r_{0} t\right) e^{-c t} t^{l} \mathrm{~d} t .
$$

They frequently appear in the potential problems with axial symmetry. How to reduce the integrals with respect to $\phi$ to the Lipschitz-Hankel type should be referred to Sasai (1991a).

The Lipschitz-Hankel integrals are expressed with the complete elliptic integrals (Eason et al., 1955), which can be expanded into the Tailor series with respect to $k^{2}=4 a r_{0} / \rho_{a}^{2}$, where

$$
\rho_{a}=\sqrt{\left(a+r_{0}\right)^{2}+\left(z-z_{0}\right)^{2}} .
$$

In the present case, $k^{2}$ is sufficiently small and even only the first term of the Tailor expansions works as a good approximation. $\Phi_{2}$ to $\Phi_{4}$ are approximated by

$$
\Phi_{2}=\frac{\pi}{2} \frac{a r_{0}}{\rho_{a}^{3}}, \Phi_{3}=\pi \frac{1}{\rho_{a}^{3}}, \Phi_{4}=\frac{3 \pi}{2} \frac{a r_{0}}{\rho_{a}^{5}} .
$$

Moreover, $\rho_{a}$ can be replaced with

$$
\rho_{0}=\sqrt{r_{0}^{2}+\left(z-z_{0}\right)^{2}}
$$

because the sensor height $z_{0}(\sim$ a few $m)$ is, usually, much larger than the borehole radius $a$ $(\sim 10 \mathrm{~cm})$. Finally, eq. (2.14) can be expressed by

$$
\begin{aligned}
& \frac{1}{C_{k}} W_{k}^{(A)}\left(\boldsymbol{r}_{0}\right)=\pi a^{2} \int_{0}^{L} \mathrm{~d} z\left[F_{A}^{(k)} \frac{x_{0}}{\rho_{0}^{3}}+F_{B}^{(k)} \frac{y_{0}}{\rho_{0}^{3}}+\right. \\
& \left.+F_{C}^{(k)} \frac{2\left(z-z_{0}\right)^{2}-r_{0}^{2}}{\rho_{0}^{5}}\right] .
\end{aligned}
$$

The first and second terms of the integrand in eq. (2.22) are horizontal dipoles in the $x$ and $y$ direction, while the third one a vertical quadrupole, respectively. The vertical quadrupole is defined as the differential of a vertical dipole in the vertical direction.

\subsection{The contribution from the circular bottom} of the borehole: $\mathrm{W}_{\mathrm{k}}{ }^{(\mathrm{B})}$

The moving point $P(x, y, z)=P(r, \theta, L)$ lies on the bottom circle and the outward normal is upward, and we find

$$
\begin{gathered}
\rho_{L}=\overline{P Q}=\left.\sqrt{r_{0}^{2}+r^{2}+\left(z-z_{0}\right)^{2}-2 r r_{0} \cos \left(\theta-\theta_{0}\right)}\right|_{z=L} \\
\boldsymbol{n}=-\mathbf{e}_{z}=(0,0,-1) .
\end{gathered}
$$

The integral over the bottom surface is given by

$$
\frac{1}{C_{k}} W_{k}^{(B)}\left(\boldsymbol{r}_{0}\right)=\int_{0}^{a} \mathrm{~d} r \int_{0}^{2 \pi}\left[F_{D}^{(k)} \frac{1}{\rho_{L}}+F_{E}^{(k)} \frac{L-z_{0}}{\rho_{L}^{3}}\right] \mathrm{d} \theta
$$

where

$$
\begin{gathered}
F_{D}^{(k)}=\left[-\frac{\mu}{3 \lambda+2 \mu} \frac{\partial u_{k}}{\partial z}-\frac{3(\lambda+\mu)}{(3 \lambda+2 \mu)} \frac{\partial u_{z}}{\partial x_{k}}+\right. \\
\left.+\frac{2(\lambda+\mu)}{3 \lambda+2 \mu} \delta_{k z} \operatorname{div} \boldsymbol{u}\right]_{z=L} \\
F_{E}^{(k)}=\left[u_{k}\right]_{z=L} .
\end{gathered}
$$

Since the radius of the bottom circle is small, the displacement at the bottom can be regarded almost identical to the one at the bottom center $(r=0, z=L) . F_{D}{ }^{(k)}$ and $F_{E}{ }^{(k)}$ are excluded from the 
integral with respect to $\theta$ and $r$; eq. (2.25) is reduced to

$$
\begin{aligned}
& \frac{1}{C_{k}} W_{k}^{(B)}\left(\boldsymbol{r}_{0}\right)=2 F_{D}^{(k)}(r=0) . \\
& \cdot \int_{0}^{a} \Phi_{1} \mathrm{~d} r+2 F_{E}^{(k)}(r=0) \int_{0}^{a} \Phi_{3} \mathrm{~d} r
\end{aligned}
$$

where

$$
\begin{gathered}
\Phi_{1}=\int_{0}^{\pi} \frac{\mathrm{d} \phi}{\rho_{L}}=\pi I(0,0 ; 0) \\
\Phi_{3}=\int_{0}^{\pi} \frac{\mathrm{d} \phi}{\rho_{L}^{3}}=\frac{\pi}{c} I(0,0 ; 1)
\end{gathered}
$$

in which

and

$$
\rho_{L}=\sqrt{R^{2}+c^{2}}
$$

$$
R=\sqrt{r_{0}^{2}+r^{2}-2 r r_{0} \cos \phi}, \quad c=L-z_{0} .
$$

The Lipschitz-Hankel integral is now defined as

$$
I(m, n ; l)=\int_{0}^{\infty} J_{m}(r t) J_{n}\left(r_{0} t\right) e^{-c t} t^{l} \mathrm{~d} t .
$$

Further integration with respect to $r$ in eq. (2.28) is not available analytically, and we are to find an approximate solution. Using the Tailor series expansion for $I(m, n ; l)$ 's, we obtain

$$
\Phi_{1}=\frac{\pi}{\sqrt{\left(r+r_{0}\right)^{2}+c^{2}}} \quad \Phi_{3}=\frac{\pi}{\left\{\left(r+r_{0}\right)^{2}+c^{2}\right\}^{3 / 2}} .
$$

The integrals in eq. (2.28) can be easily obtained as

$$
\begin{aligned}
& \int_{0}^{a} \Phi_{1} \mathrm{~d} r=\log \frac{r_{0}+a+\sqrt{\left(r_{0}+a\right)^{2}+c^{2}}}{r_{0}+\sqrt{r_{0}^{2}+c^{2}}} \\
& \int_{0}^{a} \Phi_{3} \mathrm{~d} r=\frac{1}{c^{2}}\left[\frac{a+r_{0}}{\sqrt{\left(a+r_{0}\right)^{2}+c^{2}}}-\frac{r_{0}}{\sqrt{r_{0}^{2}+c^{2}}}\right] .
\end{aligned}
$$

If the observation point is far from the borehole, i.e. $r_{0} \gg a$, eqs. ((2.35) and (2.36)) approach zero. Even near around the borehole, i.e. $r_{0} \sim 0,(2.35)$ and (2.36) become negligibly small, because usually $c \gg a$. Thus we can neglect the contribution from the bottom circle of the borehole.

\subsection{The contribution from the circular top of the borehole: $\mathrm{W}_{\mathrm{k}}{ }^{(\mathrm{C})}$}

This term comes from the counterbalance that we have to subtract the contribution from the vanishing portion of the ground surface, which is the circular outlet of the borehole with radius $a$. Now the moving point $P(x, y, z)=P(r, \theta, 0)$ is on the ground $z=0$ and the outward normal is upward, and hence

$$
\begin{gathered}
\rho_{S}=\overline{P Q}=\left.\sqrt{r_{0}^{2}+r^{2}+\left(z-z_{0}\right)^{2}-2 r r_{0} \cos \left(\theta-\theta_{0}\right)}\right|_{z=0} \\
\boldsymbol{n}=-\mathbf{e}_{z}=(0,0,-1) .
\end{gathered}
$$

We follow the same procedure as in the case of Section 2.2, and easily find a solution by putting $L=0$. Thus $W_{k}^{(c)}$ is given by

$$
\begin{aligned}
& \frac{1}{C_{k}} W_{k}^{(C)}\left(\boldsymbol{r}_{0}\right)=2 F_{G}^{(k)}(r=0) . \\
& \int_{0}^{a} \Phi_{1} \mathrm{~d} r+2 F_{H}^{(k)}(r=0) \int_{0}^{a} \Phi_{3} \mathrm{~d} r
\end{aligned}
$$

where

$$
\begin{gathered}
F_{G}^{(k)}=\left[-\frac{\mu}{3 \lambda+2 \mu} \frac{\partial u_{k}}{\partial z}-\frac{3(\lambda+\mu)}{(3 \lambda+2 \mu)} \frac{\partial u_{z}}{\partial x_{k}}+\right. \\
\left.+\frac{2(\lambda+\mu)}{3 \lambda+2 \mu} \delta_{k z} \operatorname{div} \boldsymbol{u}\right]_{z=0} \\
F_{H}^{(k)}=\left[u_{k}\right]_{z=0}
\end{gathered}
$$

$\Phi_{1}$ and $\Phi_{3}$ are defined as

$$
\begin{gathered}
\Phi_{1}=\int_{0}^{\pi} \frac{\mathrm{d} \phi}{\rho_{S}}=\pi I(0,0 ; 0) \\
\Phi_{3}=\int_{0}^{\pi} \frac{\mathrm{d} \phi}{\rho_{S}^{3}}=\frac{\pi}{c} I(0,0 ; 1)
\end{gathered}
$$

in which

$$
\rho_{S}=\sqrt{R^{2}+c^{2}}
$$

and

$$
R=\sqrt{r_{0}^{2}+r^{2}-2 r r_{0} \cos \phi}, \quad c=-z_{0}\left(z_{0}<0\right) .
$$

With the aid of approximation formulas (2.34), we can evaluate the integrals on the right hand side of eq. (2.39), which are given by eqs. 
((2.35) and (2.36)) with $c=\left|z_{0}\right|$. We find again that $W_{k}^{(C)}$ is negligibly small because $\left|z_{0}\right| \gg a$. $W_{k}^{(C)}$ may play a significant role when we observe the magnetic field very close to the surface outlet, i.e. $r \sim 0$ and $z_{0} \sim a$. In ordinary tectonomagnetic observations, however, we cannot achieve such measurements just above the ground surface because of high field gradient. Hence $W_{k}^{(C)}$ can be neglected as compared with $W_{k}^{(A)}$.

In conclusion, the piezomagnetic field produced by the existence of a borehole can be represented by the first term of the contribution $W_{k}^{(A)}$, i.e. eq. (2.22). However, we may not disregard $W_{k}^{(B)}$ and $W_{k}^{(C)}$ when we consider the effect of the casing pipe which is usually made of high $\mu$ metals. They result in additional magnetic fields near the end of the pipe: the magnetic lines of force are absorbed into the metallic wall and they spread out from the end near the ground into the air. To evaluate this effect, the approximate solutions, i.e. eqs. ((2.35) and (36)), are no longer useful, and we must numerically conduct integrations with respect to $r$ according to eq. (2.28) and eq. (2.39).

\section{A case study: the Mogi model}

Suppose that there is a spherical cavity of radius $b$ at a depth of $D$ in an elastic half-space, within which a hydrostatic pressure $\Delta P$ occurs. Such a simple model works effectively to interpret crustal deformation around volcanoes (Mogi, 1958). The piezomagnetic field produced by the Mogi model was investigated in detail by Sasai (1991a). The displacement field of the Mogi model is given by

$$
\begin{aligned}
& u_{x}=\frac{C}{2 \mu}\left\{\frac{x}{R_{1}^{3}}+\frac{\lambda+3 \mu}{\lambda+\mu} \frac{x}{R_{2}^{3}}-\frac{6 x z(z+D)}{R_{2}^{5}}\right\} \\
& u_{y}=\frac{C}{2 \mu}\left\{\frac{y}{R_{1}^{3}}+\frac{\lambda+3 \mu}{\lambda+\mu} \frac{y}{R_{2}^{3}}-\frac{6 y z(z+D)}{R_{2}^{5}}\right\} \\
& u_{z}=\frac{C}{2 \mu}\left\{\frac{z-D}{R_{1}^{3}}+\frac{(\lambda-\mu) z-(\lambda+3 \mu) D}{\lambda+\mu} \frac{1}{R_{2}^{3}}+\right. \\
& \left.-\frac{6 z(z+D)^{2}}{R_{2}^{5}}\right\}
\end{aligned}
$$

where

$$
R_{1}=\sqrt{x^{2}+y^{2}+(z-D)^{2}} \quad R_{2}=\sqrt{x^{2}+y^{2}+(z+D)^{2}}
$$

and

$$
C=-\frac{1}{2} b^{3} \Delta P .
$$

Note that the origin of the Cartesian coordinates for eqs. ((3.1) to (3.3)) is at the ground surface right above the center of the pressure source. We should rewrite eq. (2.22), of which coordinates origin is the center of the outlet of a borehole, to the one based on the new coordinates. Let the center of the top of the borehole be $\left(x_{b}, y_{b}, 0\right)$ as measured from the new origin. Then we have

$$
\begin{aligned}
& \frac{1}{C_{k}} W_{k}^{(B H)}\left(\boldsymbol{r}_{0}\right)=\pi a^{2} \int_{0}^{L} \mathrm{~d} z\left[F_{A}^{(k)} \frac{x_{0}-x_{b}}{\rho_{b}^{3}}+F_{B}^{(k)} \frac{y_{0}-y_{b}}{\rho_{b}^{3}}+\right. \\
& \left.+F_{C}^{(k)} \frac{r_{b}^{2}-2\left(z-z_{0}\right)^{2}}{\rho_{b}^{5}}\right]
\end{aligned}
$$

where

$$
\rho_{b}=\sqrt{r_{b}^{2}+\left(z-z_{0}\right)^{2}} \quad r_{b}=\sqrt{\left(x_{0}-x_{b}\right)^{2}+\left(y_{0}-y_{b}\right)^{2}} .
$$

Substituting the displacement field given by eqs. ((3.1) to (3.3)) into eqs. ((2.11) to (2.13)), we obtain

$$
\begin{aligned}
& \frac{2 \mu}{C} F_{A}^{(x)}=-\frac{3 \lambda+4 \mu}{3 \lambda+2 \mu}\left(\frac{1}{R_{1}^{3}}-\frac{3 x_{b}^{2}}{R_{1}^{5}}\right)-\frac{(3 \lambda+4 \mu)(\lambda+3 \mu)}{(3 \lambda+2 \mu)(\lambda+\mu)} \\
& \left(\frac{1}{R_{2}^{3}}-\frac{3 x_{b}^{2}}{R_{2}^{5}}\right)+\frac{6(3 \lambda+4 \mu)}{3 \lambda+2 \mu} z(z+D)\left(\frac{1}{R_{2}^{5}}-\frac{5 x_{b}^{2}}{R_{2}^{7}}\right)+ \\
& -\frac{8 \mu}{3 \lambda+2 \mu}\left(\frac{1}{R_{2}^{3}}-\frac{3(z+D)^{2}}{R_{2}^{5}}\right) \\
& \frac{2 \mu}{C} F_{A}^{(y)}=\frac{3(3 \lambda+4 \mu)}{3 \lambda+2 \mu} x_{b} y_{b}\left\{\frac{1}{R_{1}^{5}}+\frac{\lambda+3 \mu}{\lambda+\mu} \frac{1}{R_{2}^{5}}+\right. \\
& \left.-\frac{10 z(z+D)}{R_{2}^{7}}\right\} \\
& \frac{2 \mu}{C} F_{A}^{(z)}=\frac{3 \lambda+4 \mu}{3 \lambda+2 \mu} \frac{3 x_{b}(z-D)}{R_{1}^{5}}+\frac{(5 \lambda+4 \mu)(3 \lambda+5 \mu)}{(3 \lambda+2 \mu)(\lambda+\mu)} . \\
& \left(\frac{3 x_{b} z}{R_{2}^{5}}\right)+\frac{9 \lambda^{2}+23 \lambda \mu+12 \mu^{2}}{(3 \lambda+2 \mu)(\lambda+\mu)}\left(\frac{3 D x_{b}}{R_{2}^{5}}\right)-\frac{3 \lambda+4 \mu}{3 \lambda+2 \mu} . \\
& \left(\frac{30 x_{b} z(z+D)^{2}}{R_{2}^{7}}\right)
\end{aligned}
$$




$$
\begin{gathered}
F_{B}^{(y)}=F_{A}^{(x)}\left(x_{b} \leftrightarrow y_{b}\right) \\
F_{B}^{(z)}=F_{A}^{(z)}\left(x_{b} \leftrightarrow y_{b}\right) \\
\frac{2 \mu}{C} F_{C}^{(x)}=\frac{x_{b}}{R_{1}^{3}}+\frac{\lambda+3 \mu}{\lambda+\mu} \frac{x_{b}}{R_{2}^{3}}-\frac{6 x_{b} z(z+D)}{R_{2}^{5}} \\
\frac{2 \mu}{C} F_{C}^{(y)}=\frac{y_{b}}{R_{1}^{3}}+\frac{\lambda+3 \mu}{\lambda+\mu} \frac{y_{b}}{R_{2}^{3}}-\frac{6 y_{b} z(z+D)}{R_{2}^{5}} \\
\frac{2 \mu}{C} F_{C}^{(z)}=\frac{z-D}{R_{1}^{3}}+\frac{(\lambda-\mu) z-(\lambda+3 \mu) D}{\lambda+\mu} \frac{1}{R_{2}^{3}}+ \\
-\frac{6 z(z+D)^{2}}{R_{2}^{5}} .
\end{gathered}
$$

In the above, $R_{1}$ and $R_{2}$ should be read as

$$
R_{1}=\sqrt{x_{b}^{2}+y_{b}^{2}+(z-D)^{2}} \quad R_{2}=\sqrt{x_{b}^{2}+y_{b}^{2}+(z+D)^{2}} .
$$

The magnetic field components are given by differentiation of the potential (3.6) with respect to $\boldsymbol{r}_{0}\left(x_{0}, y_{0}, z_{0}\right)$

$$
\begin{aligned}
& \frac{1}{\pi a^{2} C_{k}} \Delta X^{(k)}=\int_{0}^{L} \mathrm{~d} z\left[F_{A}^{(k)}\left\{-\frac{1}{\rho_{b}^{3}}+\frac{3\left(x_{0}-x_{b}\right)^{2}}{\rho_{b}^{5}}\right\}+\right. \\
& +F_{B}^{(k)} \frac{3\left(x_{0}-x_{b}\right)\left(y_{0}-y_{b}\right)}{\rho_{b}^{5}}+F_{C}^{(k)}\left\{\frac{3\left(x_{0}-x_{b}\right)}{\rho_{b}^{5}}+\right. \\
& \left.\left.-\frac{15\left(x_{0}-x_{b}\right)\left(z-z_{0}\right)^{2}}{\rho_{b}^{7}}\right\}\right]
\end{aligned}
$$

$\frac{1}{\pi a^{2} C_{k}} \Delta Y^{(k)}=\int_{0}^{L} \mathrm{~d} z\left[F_{A}^{(k)} \frac{3\left(x_{0}-x_{b}\right)\left(y_{0}-y_{b}\right)}{\rho_{b}^{5}}+\right.$ $+F_{B}^{(k)}\left\{-\frac{1}{\rho_{b}^{3}}+\frac{3\left(y_{0}-y_{b}\right)^{2}}{\rho_{b}^{5}}\right\}+F_{C}^{(k)}\left\{\frac{3\left(y_{0}-y_{b}\right)}{\rho_{b}^{5}}+\right.$ $\left.\left.-\frac{15\left(y_{0}-y_{b}\right)\left(z-z_{0}\right)^{2}}{\rho_{b}^{7}}\right\}\right]$

$\frac{1}{\pi a^{2} C_{k}} \Delta Z^{(k)}=\int_{0}^{L} \mathrm{~d} z\left[F_{A}^{(k)} \frac{3\left(x_{0}-x_{b}\right)\left(z_{0}-z\right)}{\rho_{b}^{5}}+\right.$ $+F_{B}^{(k)} \frac{3\left(y_{0}-y_{b}\right)\left(z_{0}-z\right)}{\rho_{b}^{5}}+F_{C}^{(k)}\left\{\frac{9\left(z_{0}-z\right)}{\rho_{b}^{5}}+\right.$

$\left.\left.-\frac{15\left(z_{0}-z\right)^{3}}{\rho_{b}^{7}}\right\}\right]$.

The 1D integrations in the above can be achieved accurately by the double exponential formula ( $c f$., Sasai, 1991a).

\section{The behaviour of the magnetic field above a borehole}

Let us investigate the behaviour of the magnetic field above a borehole by assuming an appropriate model. Table I summarizes the mechanical parameters of the Mogi model, the size and the position of a borehole, together with some magnetic parameters. As for the geomag-

Table I. Parameters of the Mogi model and magnetic environments.

\begin{tabular}{ccc}
\hline \hline Parameter name & Abbr. & \\
\hline Maximum uplift & $\Delta h$ & $10 \mathrm{~cm}$ \\
Source depth & $D$ & $3 \mathrm{~km}$ \\
Rigidity & $\mu$ & $3.5 \times 10^{10} \mathrm{~N} / \mathrm{m}^{2}$ \\
Average magnetization & $J$ & $5 \mathrm{~A} / \mathrm{m}$ \\
Stress sensitivity & $\beta$ & $2 \times 10^{-3} \mathrm{MPa}^{-1}$ \\
Geomagnetic dip & $I_{0}$ & $60^{\circ}$ \\
Geomagnetic declination & $D_{0}$ & $\mathrm{~N} 16^{\circ} \mathrm{E}$ \\
\hline
\end{tabular}

Piezomagnetic field due to the Mogi model $(F)$

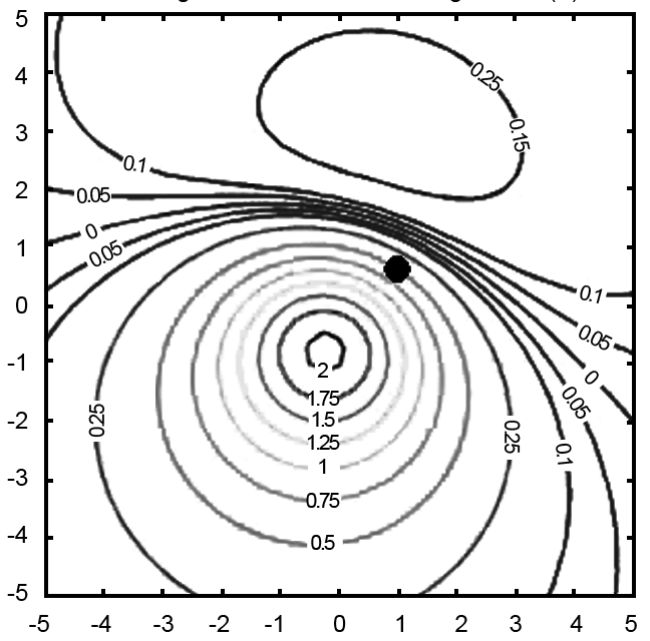

Fig. 3. Total intensity changes on the ground $(2.5 \mathrm{~m}$ above the surface) due to the Mogi model. Unit in nT. This area shows $10 \mathrm{~km} \times 10 \mathrm{~km}$ square of which center is right above the pressure source. A solid circle indicates the horizontal position of a borehole ( 2 $\mathrm{km}$ north and $1 \mathrm{~km}$ east from the center). 

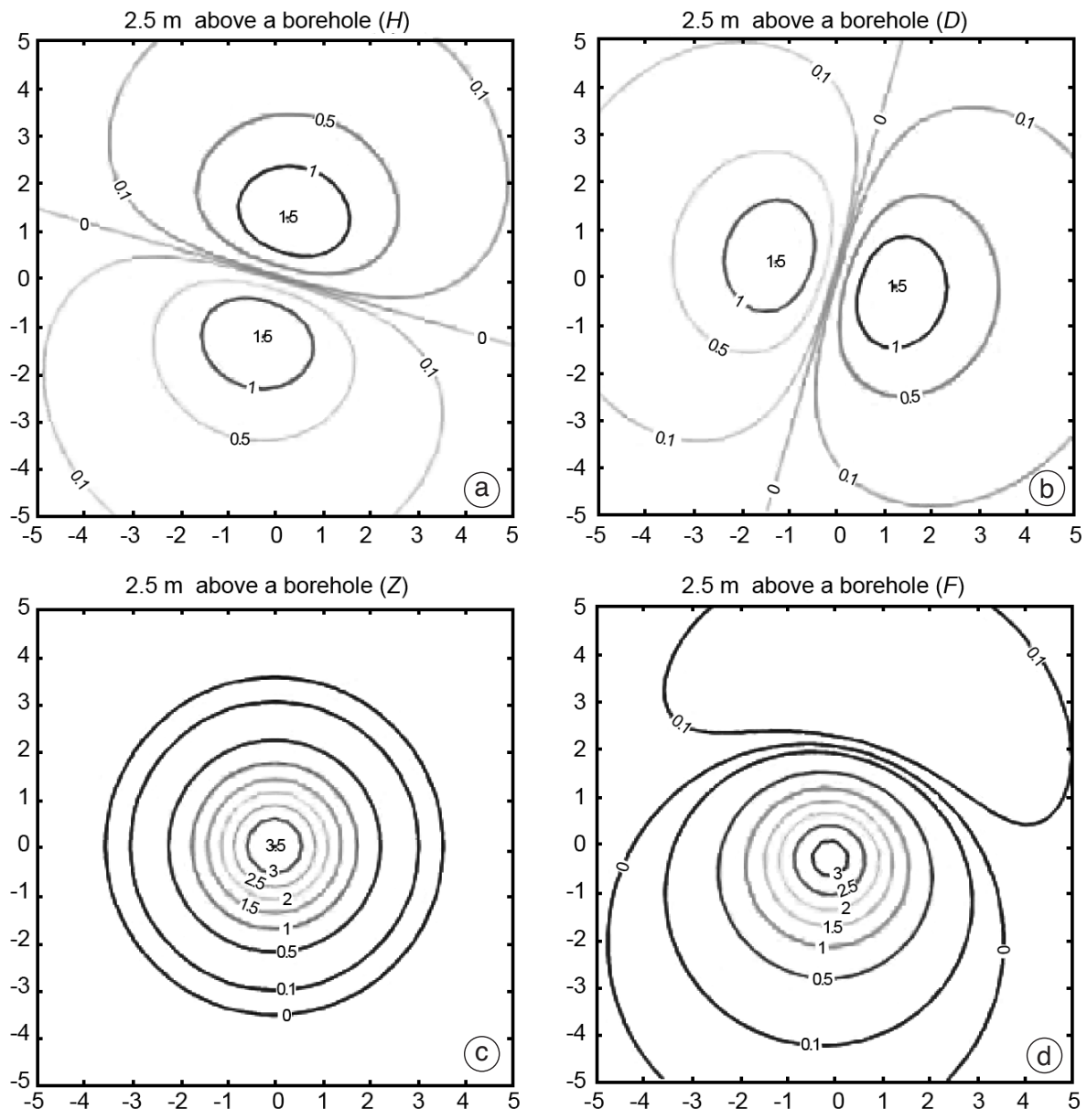

Fig. 4a-d. Four components of the piezomagnetic change due to a borehole: a) $H$, b) $D$, c) $Z$ and d) $F$ component. Each square shows $10 \mathrm{~m} \times 10 \mathrm{~m}$ area of which center is the outlet of a borehole. Unit in $\mathrm{nT}$.

netic environment, we assume the one in Long Valley Caldera. Figure 3 shows the total intensity change produced by the assumed Mogi model, in which we used Sasai's (1991a) point source solution. The magnetic field is measured at $2.5 \mathrm{~m}$ above the ground surface.

Suppose that there is a borehole for example at a point indicated by a solid circle in fig. 3 , i.e. $0.5 \mathrm{~km}$ north and $1 \mathrm{~km}$ east from the uplift center. Figure 4a-d shows the four components of the magnetic field change at a height of $2.5 \mathrm{~m}$ over $10 \mathrm{~m}$ by $10 \mathrm{~m}$ square with its center just above the borehole. Note that the effect of a borehole is extremely local, i.e. within several meters from its outlet.

As we can easily imagine from the two horizontal components and particularly from the vertical component, the magnetic lines of force spread out from the ground surface and are absorbed into the borehole. Such magnetic field corresponds to the third term on the right hand side of eq. (2.22), which is a line of vertical quadrupoles along the borehole axis. The magnetic lines of force of vertical quadrupoles ef- 
fectively come out into the air, while those of a line of horizontal dipoles encircle within the borehole. It turned out that the contribution to the external magnetic field from the vertical quadrupoles was two orders of magnitude larger than that of the horizontal dipoles.

However, even a line of vertical quadrupoles is successive arrangement of vertical dipoles
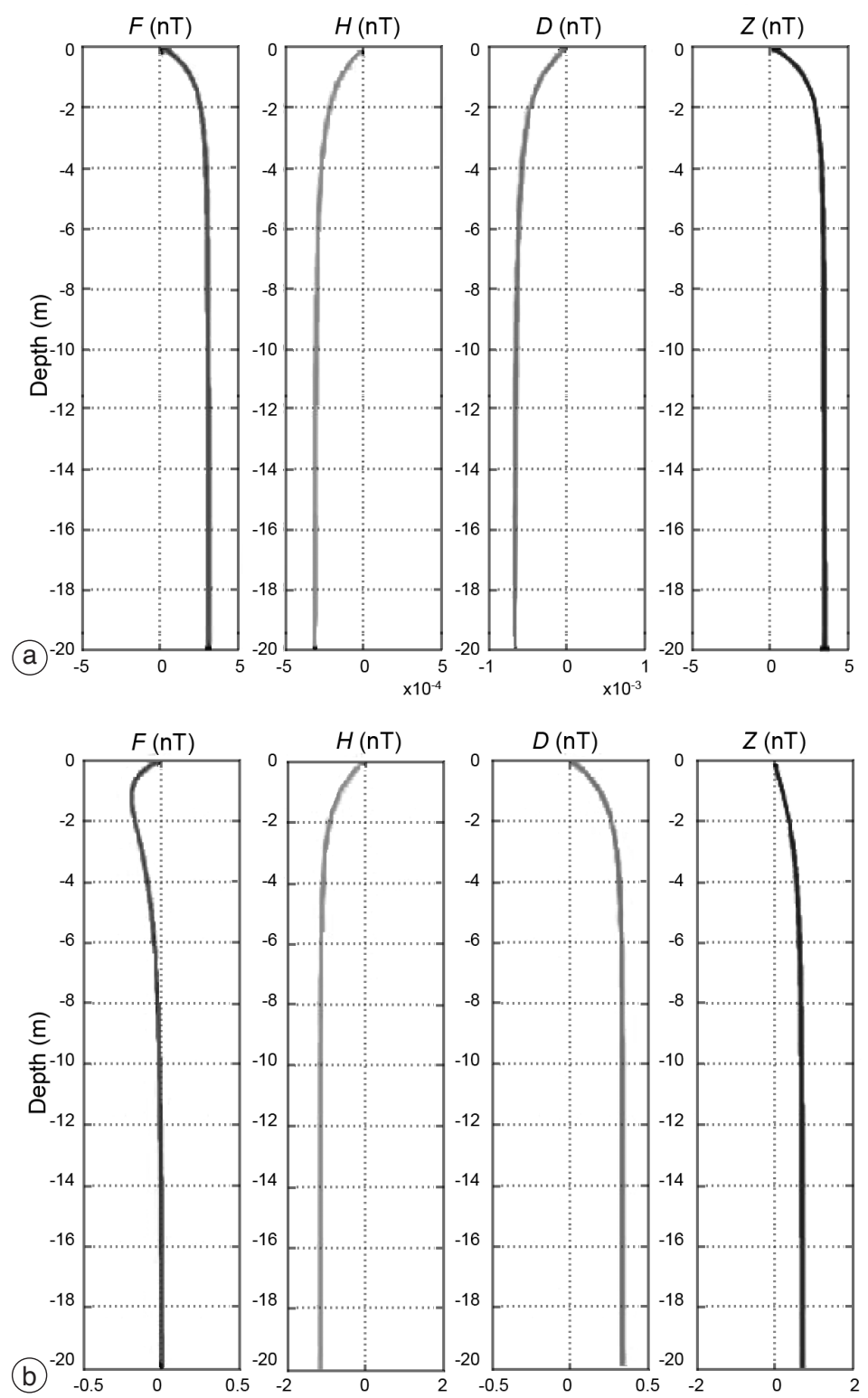

Fig. 5a,b. Depth dependence of the contribution from the cylindrical surface of a borehole: a) $2.5 \mathrm{~m}$ just above the outlet of a borehole; b) $2 \mathrm{~m}$ north and $2.5 \mathrm{~m}$ above the outlet. 



Fig. 6a,b. Changes in the total intensity above a borehole: a) $2.0 \mathrm{~m}$ above and b) $1.5 \mathrm{~m}$ above the ground surface. Unit in nT.

with opposite sign, where the magnetic field cancel with each other. The effect of deep-seated magnetic sources is difficult to reach the ground surface. Figure 5a shows cumulative changes in each component of the magnetic field at a point $2.5 \mathrm{~m}$ just above the outlet of the borehole as it elongates downward. The depth dependence of the borehole effect is not so monotonous as compared with fig. 5a at another point, which is represented by fig. 5b. This figure shows cumulative magnetic changes at a point 2 $\mathrm{m}$ north and $2.5 \mathrm{~m}$ above the borehole. The total intensity shows its maximum around $1 \mathrm{~m}$, and then it reaches the final value around $10 \mathrm{~m}$ length. Generally, the magnetic field above the ground is determined by the contribution from the shallower part of a borehole from top to a 10 $m$ length. In other words, a borehole cannot drag out the magnetic lines of force at depth. This is rather disappointing.

The influence of a borehole appears very locally around its outlet. We investigated the distribution of total intensity changes by changing the sensor height as shown in fig. 6a at a height of $2 \mathrm{~m}$ and in fig. $6 \mathrm{~b} 1.5 \mathrm{~m}$, respectively. We may expect significant amount of enhanced magnetic signals only near around the outlet of a borehole. However, in such near field, the 1st order approximation solution eq. (2.22) may not be valid, because it gives a divergent magnetic field at the top of a borehole.

\section{Discussion}

Since 1998 we have conducted continuous measurements of geomagnetic total force intensity and area survey of Self Potential (SP) in Long Valley Caldera, California, in order to detect possible changes in the EM fields associated with intrusive events at depth. Actually, Mueller and Johnston (1998) observed remarkable magnetic changes associated with reactivation of the resurgent domes in the early 1990's. At one observation site PLV, we installed two proton magnetometers, one close to a buried vertical pipe (borehole) and the other $40 \mathrm{~m}$ away in order to discriminate a sub-nanotesla change if any. An episodic event, i.e. swarm earthquakes and crustal inflation, was triggered in the caldera by the October 16, 1999, Hector Mine Earthquake of $M 7.1$ in Southern California. Figure 7 shows the simple differences in the total intensity between the two magnetometers at PLV and the volumetric strain changes at a different observation site. Total intensity difference showed gradual increase of up to $0.3 \mathrm{nT}$ from the latter half of October to early November, which is 
more or less similar to the accelerated volumetric strain changes after the Hector Mine earthquake. However, it should be noted that no such a step-like change occurred in the geomagnetic field as observed in the strain data.

Johnston et al. (2000) obtained a source model for volumetric strain changes in Long Valley Caldera which were triggered by the Hector Mine earthquake. This model involved aseismic normal faulting plus inflation, about $7 \mathrm{~km}$ WNW from PLV site, at a depth of $7 \mathrm{~km}$. The volumetric inflation source was required to explain the strain data. According to Utsugi et al. (2000), a nearly vertical dip-slip fault produces negligible piezomagnetic field, while a Mogi pressure source effectively generates an observable one (Sasai, 1991a). The observed magnetic changes could thus be produced mainly by the volume source. Hence the results shown in fig. 7 strongly motivated the present study.
Magnetic field change of any tectonic model is proportional to its moment or the intensity of the source. It greatly depends also on the depth of the source. The triggered normal faulting occurred on a slightly inclined fault of $1 \mathrm{~km}$ by $1 \mathrm{~km}$ wide with $1.4 \mathrm{~cm}$ dislocation at a depth of $7 \mathrm{~km}$. Its seismic moment is estimated as $4.9 \times 10^{19}$ dyne-cm. On the otherhand, the moment of the Mogi source given in fig. 3 is estimated as $C=6.3 \times 10^{23}$ dyne-cm. This source for the strain transient triggered by Hector Mine earthquake has a moment that is too small and is too deeply located to generate any observable magnetic change even with the aid of an enhancement effect of a borehole.

Hashimoto et al. (2003) summarized the magnetic observations in Long Valley Caldera during the period from 1999 to 2001. They investigated the cause of annual variations prominent at several stations, which were ascribed to
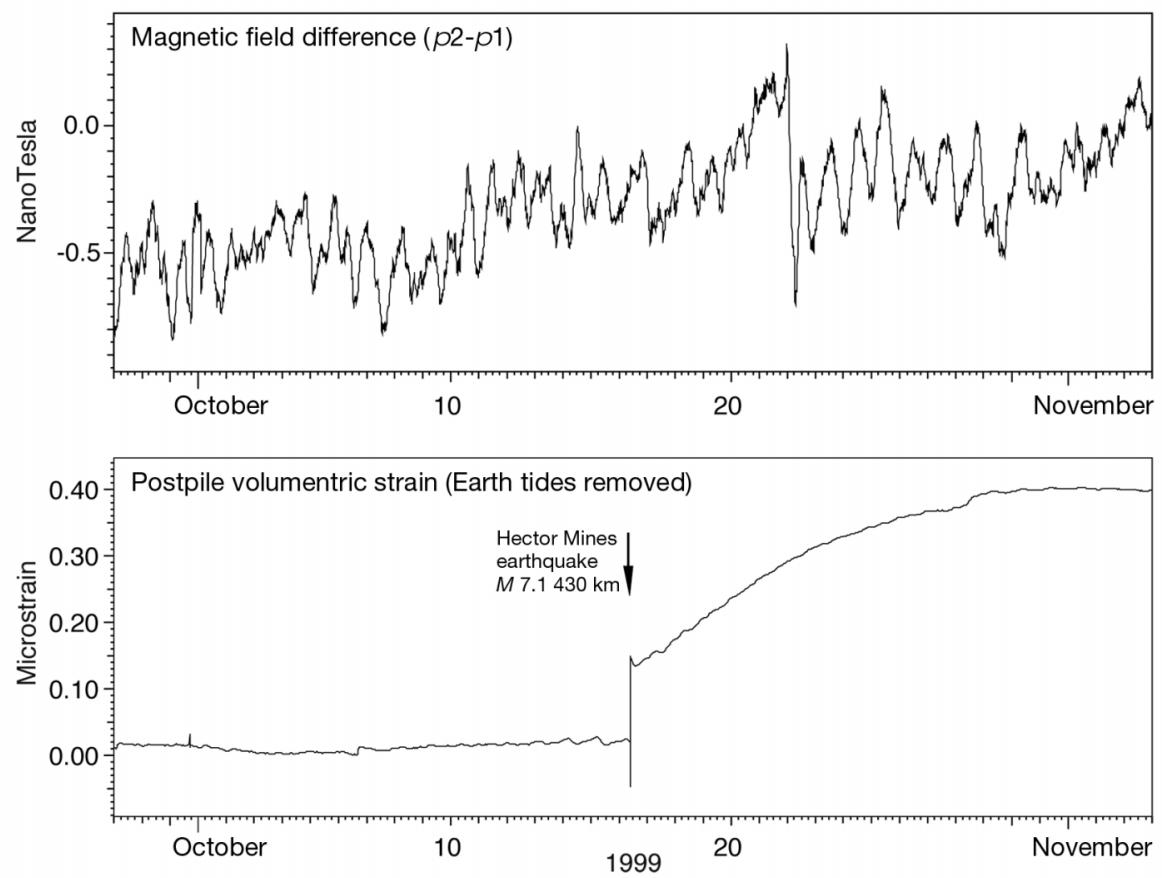

Fig. 7. Total intensity difference between two magnetometers (Unit in nT) at PLV in the upper curve and volumetric strain (Unit in $\mu$-strain) in the lower one at POP site during the period from September 28 to November 3,1999 . Total intensity and strain were measured at every $10 \mathrm{~min}$ interval. 
changes in the ground temperature (Utada et al., 2000). They successfully compensated for the annual variations on the basis of ground temperature data. Then they removed some apparent variations in the total intensity differences caused by the vectorial differences of the local main geomagnetic field as well as by the local induction effect, i.e. what is called the 3-components correction or the prediction-error filter technique. They concluded that there was no significant change larger than $0.5 \mathrm{nT}$ at PLV. As we have seen in fig. 7, the observed variation at PLV, if any, is at most $0.3 \mathrm{nT}$ or so, which does not conflict with Hashimoto et al.'s (2003) conclusion.

At PLV site in Long Valley Caldera, a vertical steel casing was buried in a nearby borehole. This produced an anomalously high field gradient, say $1000 \mathrm{nT} / \mathrm{m}$ or more. We thus installed the sensor within a few $\mathrm{m}$ of the borehole. Yamamoto (1990) conducted an experimental observation of the geomagnetic field at the bottom of a borehole using flux-gate magnetometers. He used a vinyl chloride tube to avoid some diffculties in magnetic measurements caused by high- $\mu$ metals. However, he reported some steps in the magnetic data associated with small felt earthquakes most probably caused by movement of the sensor. Although the most up-to-date Overhauser type proton magnetometer works under a relatively high field gradients, only a few mm displacement of a sensor would result in an apparent variation. It would then be difficult to discriminate signals of piezomagnetic origin.

An optimum arrangement for geomagnetic observations using a borehole would be a) a borehole of several hundreds meters length plus; b) a spherical cavity of radius 1 to $2 \mathrm{~m}$ in order to avoid the high field gradient at the bottom of the borehole. The magnetometer sensor is placed at the center of the cavity, which is fixed with silica or any non-magnetic substance. We don't use the casing pipe of high $\mu$ metal for the protection of the borehole. The magnetic field at any position in such a system, even inside the borehole, can be represented rigorously by eqs. ((2.14), (2.28) and (2.39)) with the aid of Lipschitz-Hankel integrals. The same procedure is applicable to evaluate the effect of the spherical cavity at the bottom of the borehole. Our next subject is to investigate the behaviour of the tectonomagnetic field under such an observation system.

\section{Acknowledgements}

We are greatly indebted to Drs. Ciro Del Negro and Antonio Meloni who carefully read the manuscript and gave us useful comments to improve it.

\section{REFERENCES}

EAson, G., B. Noble and I.N. SNEdDON (1955): On certain integrals of Lipschitz-Hankel type involving products of Bessel functions, Phil. Trans. R. Soc. London Ser. A, 247, 529-551.

Hashimoto, T., Y. TANAKA, M.J.S. JohnStOn, M. UtSUGi, Y. SASAI and S. SAKANAKA (2003): On the annual variations in geomagnetic differences observed in Long Valley Caldera, California, Ann. Disas. Prev. Res. Inst., Kyoto Univ., 46B, 765-777 (in Japanese with English abstract).

Johnston, M.J.S., D.P. Hill and A.M. PITT (2000): Strain transient recorded in the Long Valley Caldera during triggered seismicity from the October 16, 1999, M 7.1 Hector Mine, California, earthquake, Eos, Trans. Am. Geophys. Un., 81, WP1384.

Mogi, K. (1958): Relations between the eruptions of various volcanoes and the deformations of the ground surfaces around them, Bull. Earthqake Res. Inst., Univ. Tokyo, 36, 99-134.

Mueller, R. and M.J.S. Johnston (1998): Review of magnetic field monitoring near active faults and volcanic calderas in California: 1974-1995, Phys. Earth Planet. Inter., 105, 131-144.

SASAI, Y. (1983): A surface integral representation of the tectonomagnetic field based on the linear piezomagnetic effect, Bull. Earthq. Res. Inst., Univ. Tokyo, 58, 763-785.

SASAI, Y. (1991a): Piezomagnetic field associated with the Mogi model revisited: analytic solution for finite spherical source, J. Geomag. Geoelectr., 43, 21-64.

SASAI, Y. (1991b): Tectonomagnetic modeling on the basis of the linear piezomagnetic effect, Bull. Earthquake Res. Inst., Univ. Tokyo, 66, 585-722.

SASAI, Y. (1994): Enhancement of piezomagnetic signals within a borehole, in Electromagnetic Phenomena Related to Earthquake Prediction, edited by M. HAYAKAWA and Y. FuJINAwA (Terra Scientific Publishing Co., Tokyo), 51-54.

Utada, H., M. NeKi and T. Kagiyama (2000): A study of annual variations in the geomagnetic total intensity with special attention to detecting volcanomagnetic signals, Earth Planets Space, 52, 91-103.

UTSUGI, M., Y. NisHIDA and Y. SASAI (2000): Piezomagnetic potentials due to an inclined rectangular fault in a semi-infinite medium, Geophys. J. Int., 140, 479-492.

Yамамото, T. (1990): Geomagnetic three components observations using a borehole, in Proceedings of Conductivity Anomaly Symposium, 187-194 (in Japanese). 